\title{
Disminución de la tasa de operación cesárea en la Fundación Clínica Valle del Lili
}

\author{
Edgard Cobo C.*
}

"La independencia del dominio español no nos puso a salvo de la demencia" Gabriel García Márquez La soledad de América Latina Conferencia Nobel.1982

\section{RESUMEN}

Hace seis años venimos trabajando en la búsqueda de intervenciones que nos permitan disminuir las altas tasas de operación cesárea en más bajas entre las clínicas privadas de la ciudad y reducciones significativas en indicaciones como cesárea previa y dist ocia diagnosticada durante el embarazo. El último período analizado, 1998-1999. corresponde a la apertura de nuestro Servicio a las madres usuarias del Plan Obligatorio de Salud (POS) y a la inserción de dos intervenciones: la atención del parto normal por médicas generales y la discusión semanal con los especialistas institucionales de las indicaciones de las cesáreas realizadas. La tasa de cesárea mostró una reducción significativa desde 67.8\% a 30.0\%, siendo más baja en las usuarias del POS (27.1\%) que en las usuarias de sistemas privados (MPP) de atención (57.4\%). Con la excepción de la cesárea iterativa, que aumentó significativamente en las usuarias de MPP, se observaron disminuciones significativas en la práctica de cesáreas primarias, distocia, sufrimiento fetal agudo y presentación podálica. Se observó que la cesárea por solicitud materna aumentó en el grupo de usuarias de MPP y no ocurrió en el de pacientes del POS. No sabemos cual es el impacto de las intervenciones descritas, ya que se requeriría un estudio clínico aleatorizado costoso y de muy difícil realización, pero se hace una amplia discusión de los resultados obtenidos después de introducirlas.

PALABRAS CLAVES: Cesárea médicamente innecesaria, tasas, disminución.

\section{SUMMARY}

In recent years a large number of medicalIy unnecesary cesarean sections have being performed alI over the country. At the Fundación Clínica Valle del Lili, a private medical center in Cali, Colombia, a c-section rate of $66.2 \%$ was registered during the first 2 years of operation (1994-95), which increased to $67.8 \%$ in 1997-98. In 1998-99, attempts to lower this extremely high rates were addressed. Three strategies were implemented to achieve this goal: a) to alIow patients from the national health system to get access to our service, b) to delegate the management of labor and delivery to female general practitioners, and c) to have a weekly group discussion of alI c-sections performed by the staff. As a result, c-section rate decreased to $30.0 \%$ (27.1\% for the patients coming from the national system and $57 \%$ for private patients) A significant reduction was observed in the 4 more frequent indications: previous c-section, dystocia, fetal distress and breech presentation. C-sections performed at patient's request showed a rate of $0.9 \%$, all of them coming from the private patients. The importance of this findings is discussed.

KEY WORDS: MedicalIy unnecesary cesarean, rates, decrease.

\section{Introducción}

Hace dos años publicamos las tasas de operación cesárea en nuestra institución, comparando dos períodos distintos de trabajo en el Departamento de Obstetricia y Ginecología (1). Desde hace más de una década, nos ha preocupado tanto la existencia en nuestro medio de tasas de cesárea que podrían figurar entre las más altas del mundo, como la relevancia que ha adquirido la práctica de la cesárea médicamente injustificada. Es así como, cuando las tasas universales oscilan entre 4 y $29 \%(2,3,4,5,6,7,8)$, en Cali oscilan entre $20 \%$ en un hospital universitario y alrededor del $85 \%$ en algunas clínicas privadas (1). Destacamos el hecho de no haber encontrado en la literatura médica universal, una sola publicación que informe tasas tan altas de operación cesárea. En nuestra institución existe una política conservadora que privilegia el parto natural; sin embargo, en los primeros años, cuando la práctica 
profesional era exclusivamente particular, resultó casi imposible desarrollar esta tendencia. Entonces, nuestra tasa de operación cesárea fue de $66.2 \%$ entre 1994 y 1995, Y aumentó a $67.8 \%$ entre 1996 y 1997. Sin embargo, era más baja que en las otras clínicas privadas de la ciudad, hecho éste que entendimos como una posibilidad esperanzadora para obtener mejores resultados futuros.

En 1998 se implementó en nuestro Departamento la atención de las pacientes dentro del Sistema de Seguridad Social en Salud establecido por la Ley 100 de 1993, que integra la salud pública, el sistema de seguridad social y los servicios de atención privada. Esta reforma, a pesar de todos los desajustes que ha producido en el ejercicio profesional de la medicina, tiene como punto a su favor el hecho de democratizar la atención médica. Desafortunadamente, la versión nacional de esta democratización es típicamente desorganizada y por demagógica, falta de recursos, razones que nos han obligado a realizar ingentes esfuerzos para garantizar una aceptable calidad de atención a las usuarias de ese sistema. Además agregamos otras 2 intervenciones: La atención de los partos normales por médicas generales y la discusión semanal de las indicaciones de las cesáreas realizadas en la Clínica. Así, hemos logrado una reducción significativa de las tasas de operación cesárea en todas nuestras pacientes, pero mucho mayor en las usuarias del Plan Obligatorio de Salud (POS), que entre las usuarias de planes de atención privada o prepagada (MPP).

Este artículo describe la magnitud de estos cambios y algunas de las características de los mismos, observadas al comparar los dos períodos anuales descritos previamente (1), con el último período anual estudiado

\section{Pacientes y Métodos}

El estudio se llevó a cabo en el Servicio de Partos de la Unidad Materno Infantil de la Fundación Clínica Valle del Lili, en la ciudad de Cali. Se comparó una cohorte de pacientes atendidas entre el $1^{\circ}$, de septiembre de 1998 y el 31 de agosto de 1999, con dos cohortes estudiadas previamente en períodos anuales entre 1994 y 1995, Y entre 1996 y 1997. Entre el primero y el segundo período estudiados, el único cambio ocurrido fue el reemplazo de la mitad del cuerpo médico del Servicio. Entre el segundo y el tercer periodos, se establecieron tres intervenciones nuevas, a saber: a) la apertura de la atención obstétrica a las usuarias del POS, b) la discusión semanal con todo el grupo de especialistas de la indicación cada una de las cesáreas practicadas tanto en pacientes del POS como en las pacientes usuarias de planes de MPP y, c) la atención del trabajo de parto y el parto por médicas generales, bajo la supervisión permanente del cuerpo de especialistas de dedicación exclusiva, quienes además realizaron todas las intervenciones quirúrgicas.

Durante el primer período se atendieron 417 nacimientos, durante el segundo, 339 y durante el tercero, 1300. En la cohorte correspondiente al tercer período se estratificó a las pacientes atendidas como usuarias de MPP, para compararlas con las usuarias del POS.

Se utilizó el mismo formulario aplicado a las pacientes que culminaron el embarazo por cesárea en los dos

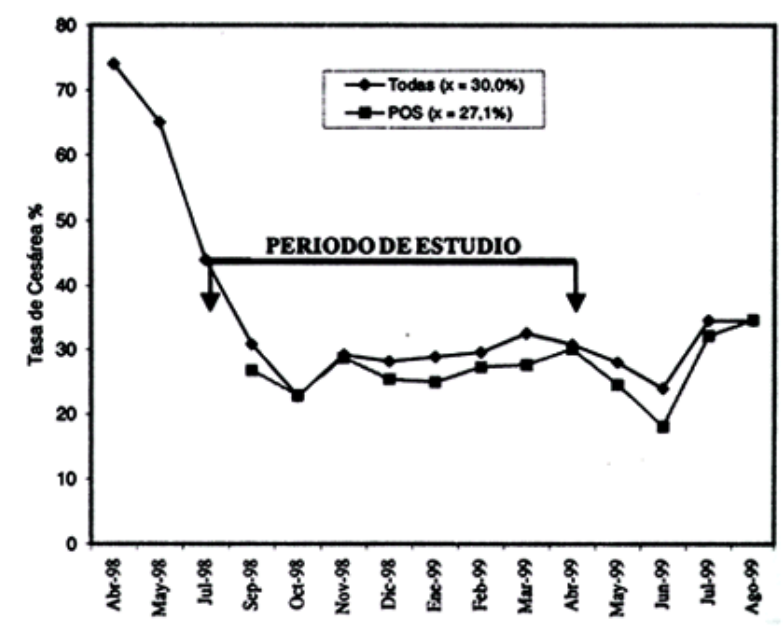

Figura 1

TASAS DE CESÁREA ANTES Y DURANTE LA IMPLEMENTACIÓN DE LAS INTERVENCIONES DISEÑADAS PARA. EL TERCER PERÍODO DE ESTUDIO. OBSÉRVESECOMOLA TASA CORRESPONDIENTEA LAS PACIENTES DEL POS ES MENOR QUE LA DE TODAS LAS PACIENTES; SIN EMBARGO,SUDISMINUCIÓNSIGUE LA MISMA TENDENCIA.

primeros períodos, el cual fue llenado por el autor tan pronto se realizaba una intervención. La información así obtenida fue llevada a una de las bases de datos de nuestra Unidad. Las variables primarias de salida fueron: la tasa de operación cesárea y la tasa de cada indicación, aceptando inicialmente como cierto el diagnóstico anotado por el médico responsable de la atención de la paciente y cambiándolo solo cuando se consideró inconsistente, de acuerdo con la evidencia aportada en la discusión semanal. Las variables secundarias fueron: gravidez, paridad, peso, edad y talla maternas, edad gestacional en el momento de la intervención, día de la semana, hora del día, momento de la cesárea respecto del trabajo de parto (anteparto eintraparto), peso del neonato, Indice de Apgar y admisión a la Unidad de Cuidado Intensivo Neonatal (UCIN).

Los datos se analizaron utilizando el programa Epiinfo 6 de la Organización Mundial de la Salud. Los resultados se presentan como tablas de distribución de frecuencias, tasas, riesgos relativos (RR), con sus respectivos intervalos de confianza del 95\% (IC95\%) y valores promedio, acompañados del error estándar de la media. La significación estadística de las diferencias se calculó con la prueba t de Student, la prueba exacta de probabilidad de Fisher y la de Chi cuadrado con factor de corrección de Yates. La significación estadística de las diferencias se estableció en un valor de $\mathrm{p}<0.05$.

El proyecto y la aplicación del formulario fueron aceptados por el Comité de Investigación de la Clínica. Las pacientes sometidas a cesárea firmaron el mismo protocolo de consentimiento informado que se estila en la institución, para todas las intervenciones quirúrgicas. 


\section{CARACTERÍSTICAS DEMOGRÁFICAS Y CLIINICAS DE LAS MADRES Y LOS RECIÉNNACIDOS}

\begin{tabular}{|l|c|c|c|}
\hline & Cohorte 1 & Cohorte 2 & Cohorte 3 \\
\cline { 2 - 4 } & $1994-1995$ & $1996-1997$ & $1998-1999$ \\
\hline Edad Materna (años) & $\mathbf{2 9 . 5} \pm 0.30 *$ & $\mathbf{2 9 . 3} \pm 039$ & $\mathbf{2 8 . 3} \pm 0.30$ \\
\hline Talla Materna (metros) & $\mathbf{1 . 6 3} \pm 0.05$ & $\mathbf{1 . 6 0} \pm 0.01$ & $\mathbf{1 . 6 2} \pm 0.02$ \\
\hline Peso Materno (kg) & $\mathbf{7 0 . 9} \pm 0.35$ & $\mathbf{7 1 . 1} \pm 1.52$ & $\mathbf{7 0 . 2} \pm 0.61$ \\
\hline Gravidez & $\mathbf{1 . 9 3} \pm 0.06$ & $\mathbf{1 . 7 4} \pm 0.02$ & $\mathbf{2 . 1} \pm 0.05$ \\
\hline Paridad & $\mathbf{0 . 2 5} \pm 0.04$ & $\mathbf{0 . 1 5} \pm 0.03$ & $\mathbf{0 . 4 8} \pm 0.06$ \\
\hline Edad Gestacional (semanas) & $\mathbf{3 8 . 4} \pm 0.15$ & $\mathbf{3 7 . 6} \pm 0.19$ & $\mathbf{3 7 . 6} \pm 0.20$ \\
\hline Peso Recién Nacidos (gr) & $\mathbf{3 1 5 7} \pm 34.2$ & $\mathbf{3 0 1 1} \pm 46.5$ & $\mathbf{3 0 2 0} \pm 42.4$ \\
\hline Apgar < 7 al minuto & $\mathbf{1 5}$ & $\mathbf{1 7}$ & $\mathbf{5 3}$ \\
\hline Apgar < 7 a los 5 minutos & $\mathbf{2}$ & $\mathbf{5}$ & $\mathbf{2 1}$ \\
\hline UCIN (número de ingresos) & $\mathbf{2 0}$ & $\mathbf{3 1}$ & $\mathbf{5 6}$ \\
\hline
\end{tabular}

* Valor promedio \pm SEM

\section{Resultados}

Cuando se compararon las características clínicas de la segunda y tercera cohortes estudiadas, se encontró una disminución estadísticamente significativa en la variable edad materna, $\mathrm{p}=0.04$, y un aumento en la gravidez, $\mathrm{p}=0.0001$, la paridad, $\mathrm{p}=0.00002$ y la admisión a la UCIN, $\mathrm{p}=0.000000$. Las demás comparaciones no mostraron diferencias estadísticamente significativas (Cuadro 1).

La tasa global de cesárea disminuyó significativamente desde $67.8 \%$ a $30.0 \%$ en el período 1998-99, con un riesgo relativo de 0.44 (IC95\%, 0.40-0.49) ( $\mathrm{p}=0.000000) . \mathrm{Al}$ separar a las pacientes de MPP de las del POS, se encontró una tasa de 57.4\%, RR: 0.85 (IC95\%, 0.72-1.00) para las primeras, la cual es significativamente menor que la del período anterior estudiado entre 1996 y 1997 ( $\mathrm{p}=$ 0.04386), que fue de $67.8 \%$. La tasa de cesárea para las pacientes del POS fue $27.1 \%$, que no se compara con el período anterior, ya que en esa época sólo atendíamos pacientes provenientes de MPP. En la figura 1, puede verse la evolución de estas tasas en ambos grupos de pacientes desde septiembre de 1998, hasta agosto de 1999.

La cesárea previa, fue la indicación más frecuente. Esta indicación ha venido presentando una consistente disminución durante los 3 períodos estudiados, así: $35.9 \%$ (148/417) durante el primer período, 28.3\% (50/339) durante el segundo y $10.1 \%(139 / 1.300)$ durante el tercero. La comparación entre las tasas de los 2 primeros períodos no resultó estadísticamente significativa $(\mathrm{p}=0.2446)$, pero si lo fueron las diferencias entre el segundo y el tercer periodos, RR: 0.65(IC95\%, 0.51-0.93), p= 0.0214 (Figuras 3 y 4), y más aún entre el primero y el tercero, RR: $0.42(\mathrm{IC} 95 \%, 0.31-0.55), \mathrm{p}=$ 0.000000 .
La distocia, fue la segunda indicación más frecuente. Presenta disminuciones significativas en los tres periodos, así: $17.0 \%(71 / 417)$ durante el primer período, $10.4 \%$ $(35 / 339)$ durante el segundo y $10.1 \%$ (132/1.300) durante el tercero. Tanto las diferencias entre los periodos 1 y 2 , RR: 0.61 (IC95\%, 0.42-0.89), como entre los periodos 2 y 3, RR: 0.54 (IC95\%, 0.36-0.79), fueron estadísticamente significativas con valores de $\mathrm{p}=0.008$ y 0.002 , respectivamente. (Figuras 3 y 4 )

El sufrimiento fetal, la tercera indicación más frecuente, se mantuvo sin variaciones entre el primero y el segundo períodos (11.6\% y $11.3 \%$, respectivamente), pero disminuyó significativamente a 10.5\%, RR: 0.41 (IC95\%, 0.260.66), $\mathrm{p}=0.0003$, entre el segundo $\mathrm{y}$ el tercer período. La presentación podálica tuvo tasas bajas, de $4.2 \%$ (14/339) para el segundo período y de $2.5 \%$ para el tercero, pero esta diferencia no alcanzó significación estadística, RR: $0.60 \quad$ (IC95\%, 0.32-1.10), $\quad \mathrm{p}=0.142$. (Figuras 3 y 4)

La solicitud materna como causa de operación cesárea tuvo una tasa global de $3.1 \%$ (12/390). Sin embargo, al estratificar la muestra por sistema de prestación del servicio, no se consignó ninguna solicitud materna en las pacientes del POS, en contraste con un 16.2\% (12/74), encontrado en las MPP. Este dato muestra que entre estas últimas pacientes la cesárea a solicitud materna, fue la segunda indicación más frecuente, después de la cesárea previa.

La cesárea primaria, tuvo una tasa global de $55.1 \%$ (230/417) durante el primer periodo y aumentó hasta 58.9\% (200/339) el segundo, para disminuir hasta $10.7 \%(139 / 1300)$ en el tercer periodo. Esta última diferencia fue estadísticamente significativa, RR: 0.18 (IC95\%, 0.15-0.22), $\mathrm{p}=0.000000$ (Cuadro 2 y Figuras 3 y 4). 


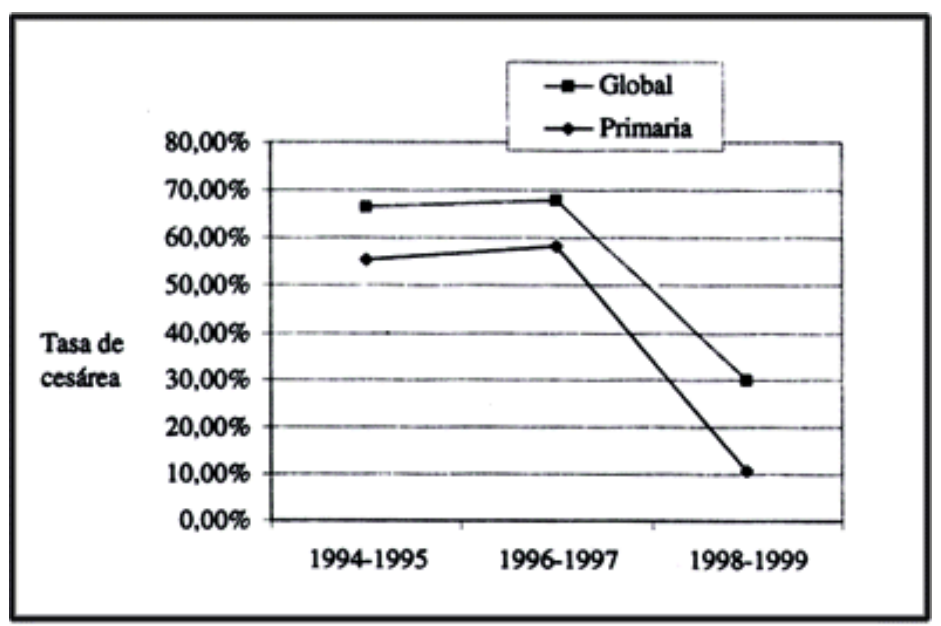

Figura 2

\section{DISMINUCIÓN DE LA TASA GLOBAL DE CESÁREA Y dE LA DE CESÁREA PRIMARIA EN LA TERCERA COHORTE.}

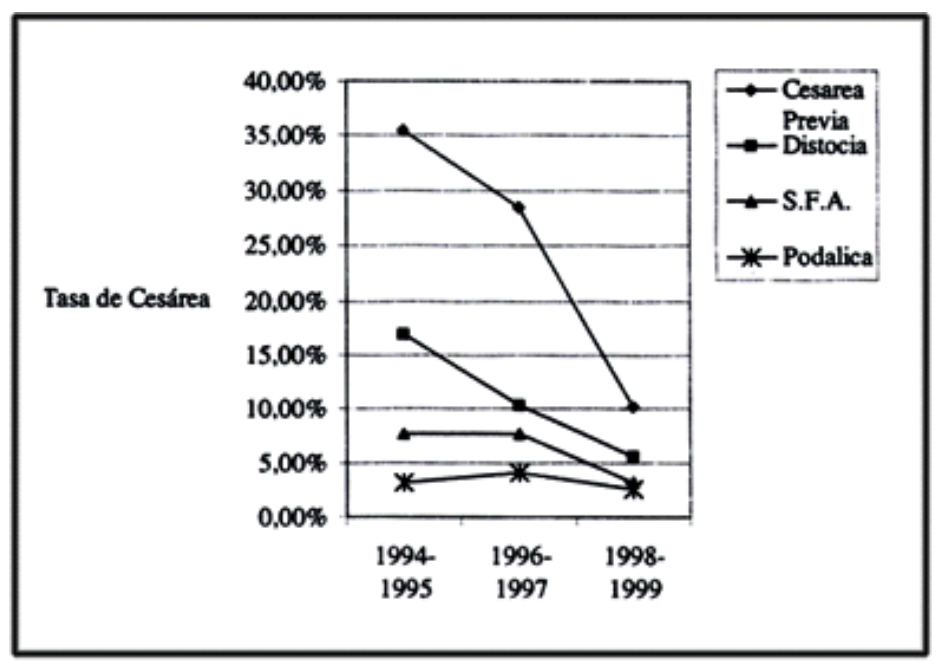

\section{Figura 3 \\ DISMINUCIÓN DE LAS TASAS \\ DE CESÁREAS EN LAS CUATRO INDICACIONES MAS FRECUENTES.}

El diagnóstico de distocia realizado durante el embarazo, o en la fase latente del parto, una situación de muy difícil verificación clínica, fue muy frecuente en el grupo de pacientes intervenidas durante el primer período de funcionamiento de nuestro servicio, con una tasa de 55.1\% (31/36), que disminuyó a $39.1 \%$ (9/23) en el segundo período y continuó disminuyendo hasta llegar a $11.9 \%(13 / 109)$ en el tercero. La disminución observada en este último período fue estadísticamente significativa, RR: 0.18 (IC95\%, 0.15- 0.22), p=0.000000 (Cuadro 2 y Figuras 3 y 4 )

Las demás indicaciones de cesárea constituyeron el $25 \%$ y no se diferencian de las descritas en nuestra publicación anterior (1), razón por la cual no las describimos aquí. Tanto los días de la semana, como las horas del día en las cuales se practicaron las intervenciones, no mostraron diferencias significativas entre los tres períodos. (Datos no mostrados en este artículo).

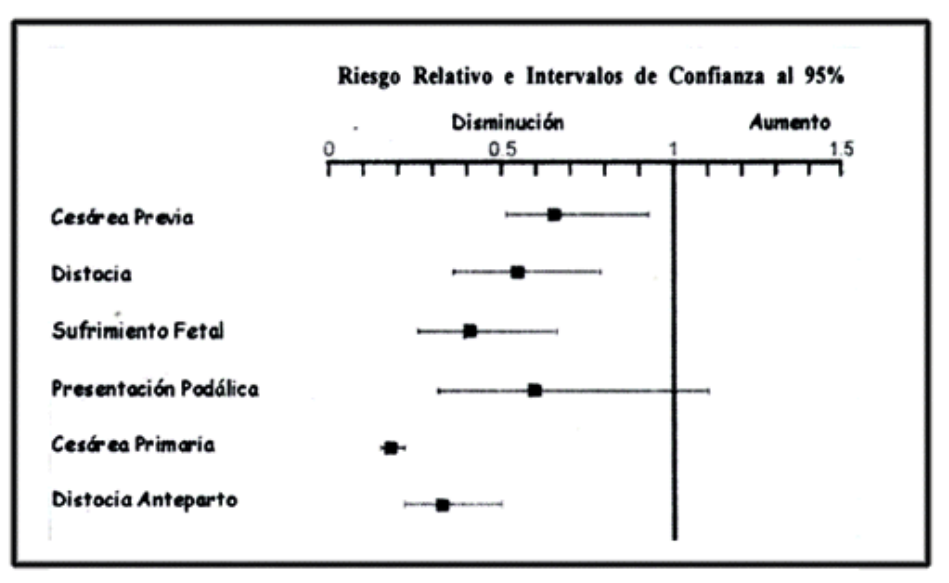

Figura 4

CAMBIOS EN LAS TASAS DE CESÁREA ENTRE EL SEGUNDO Y EL TERCER PERÍODO (1996-97 Y 1998-99). SI TOMAMOS COMO EJEMPLO LA CESÁREA PRIMARIA VEMOS COMOTUVO UN RR DE 0,18(IC95\%, 0,15 - 0,22), O SEA UNA DISMINUCIÓN DEL 82\%, ESTADÍSTICAMENTE SIGNIFICATIVA $(P=0,000000)$. LAS DEMÁS VARIABLES PRESENTADAS EN EL GRÁFICO MUESTRAN TAMBIÉN DISMINUCIONES ESTADÍSTICAMENTE SIGNIFICATIVAS, CON LA EXCEPCIÓN DE LA PRESENTACIÓN PODÁLICA QUE NO ALCANZA A TENER ESE NIVEL.

El análisis de las tasas de cesárea por indicación, realizado separadamente en las pacientes de MPP muestra una tendencia a la disminución comparable a las pacientes del POS, aunque solo tienen significación estadística las medidas correspondientes a la tasa de cesárea por cesárea previa, que es la única que aumenta, RR: 1.68 (IC95\%, 1.13-2.50), p=.0154, y la cesárea primaria, que disminuye marcadamente, RR: 0.43 (IC95\%, 0.32-0.59), $\mathrm{p}=0.0000000$ (Figura 5). Las cifras globales muestran diferencias apreciables en las indicaciones de cesárea previa y solicitud materna, pero son bastante similares en las otras, como se aprecia en el Cuadro 3. Para efectos de comparación, se agregan a ese cuadro las tasas que para esas indicaciones han sido publicadas por el Hospital del Condado de Jacksonville, EE.UU, en donde existe una política oficial de disminución de las tasas de cesárea basada en la enseñanza y la práctica rigurosas de la clínica obstétrica (8).

En cuanto hace relación a los resultados perinatales, las variables de salida estudiadas mostraron que el número de recién nacidos con Indice de Apgar menor de 7 al minuto y a los 5 minutos, no varió significativamente, $\mathrm{p}=0.5420$ y 0.9253 , respectivamente y que aunque, como es lógico, aumentó el número de admisiones a la Unidad de Cuidado Intensivo Neonatal (UCIN), su proporción se redujo significativamente en un 67\%, RR: 0.33 (IC95\%, 0.22-0.50), p= 0.000000. (Figura 3).

\section{Discusión}

El resultado más importante encontrado en este estudio fue la reducción en las tasas de operación Cesárea, registradas después de establecer los cambios e intervénciones diseñadas para el tercer período. En ese resultado debe destacarse también la disminución de la cesárea 
Cuadro 2

VARIACIONES EN LAS TASAS DE CESÁREA, POR INDICACIÓN, ASÍ COMO EN LA PRÁCTICA DE CESÁREA PRIMARIA Y DIAGNOSTICO DE DISTOCIA REALIZADO DURANTE EL EMBARAZO Y EN LA FASE LATENTE DEL PARTO

\begin{tabular}{|l|c|c|c|}
\hline & Cohorte 1 & Cohorte 2 & Cohorte 3 \\
\hline & $1994-1995$ & $1996-1997$ & 339 \\
\hline Nacimientos & 417 & 300 \\
\hline Partos & $33.8 \%(141 / 417)$ & $32.2 \%(109 / 339)$ & $70 \%(910 / 1300)$ \\
\hline Cesáreas & $66.2 \%(276 / 417)$ & $67.8 \%(276 / 417)$ & $30.0 \%(390 / 1300)$ \\
\hline Cesárea Previa & $35.5 \%(148 / 417)$ & $28.3 \%(96 / 339)$ & $10.2 \%(132 / 1300)$ \\
\hline Distocia (DCP) & $17 \%(71 / 417)$ & $10.3 \%(35 / 339)$ & $3.5 \%(72 / 339)$ \\
\hline Sufrimiento Fetal & $7.7 \%(32 / 417)$ & $7.7 \%(26 / 339)$ & $3.1 \%(41 / 1300)$ \\
\hline Presentación Podálica & $3.1 \%(13 / 417)$ & $4.1 \%(14 / 339)$ & $2.5 \%(32 / 1300)$ \\
\hline
\end{tabular}

\begin{tabular}{|l|c|c|c|}
\hline Cesárea Primaria & $55.1 \%(230 / 417)$ & $58.9 \%(200 / 339)$ & $10.7 \%(139 / 1300)$ \\
\hline Distocia en Cesárea Primaria & 36 & 23 & 109 \\
\hline Diagnóstico anteparto & $86.1 \%(31 / 36)$ & $39.1(9 / 23)$ & $11.9 \%(13109)$ \\
\hline
\end{tabular}

primaria y la de la cesárea por distocia diagnosticada antes de la iniciación del trabajo de parto.

La notable disminución de la tasa de cesárea primaria es una cifra no solo estadísticamente significativa, sino también clínicamente significativa, como quiera que un cambio de esa magnitud se refleja sin ninguna duda, y como es obvio, en las tasas de cesárea iterativa. La significación clínica de los resultados es un hecho que usualmente no se tiene en cuenta cuando una diferencia cualquiera resulta menor de 0.05 , lo cual nos lleva a ignorar su significación clínica, en ocasiones mucho más importante que la primera. Bien conocidas son las incontables significaciones estadísticas que al ser reproducidas con estudios mejor diseñados perdieron su significación y de muchas otras que a pesar de serlo, no tuvieron la menor importancia clínica $(9,10,11)$.

En nuestro artículo anterior (1), describimos un aumento en la tasa de cesárea primaria, observado entre los dos primeros períodos de estudio. Este hecho inesperado pudo deberse a que el cambio de algunos especialistas durante el segundo período, se hizo con elementos más jóvenes, que resistieron al comienzo la política conservadora de la institución, para ir aceptándola posteriormente, como resultado de las reuniones semanales para la discusión de los casos, en las cuales nunca exigimos el cumplimiento de normas por imposición, sino por convicción. Así vimos como la reducción en la tasa de cesárea primaria no sólo ocurrió en el grupo de pacientes del POS, sino también, aunque en menor grado, en las MPP. Existen estudios publicados que muestran las bondades de la política de reducción de las tasas de cesárea por convicción, más que por mandato $(12,13)$

Frente al aumento en la cesárea primaria observado en el segundo período, calculamos hace dos años un ritmo de crecimiento anual de $4 \%$ en esta categoría de intervención, destacando que si ese ritmo se sostenía en el tiempo, el parto natural desaparecería en las clínicas privadas de la ciudad en el muy breve lapso de 4 años, una posibilidad realmente vergonzosa. Para nuestra fortuna, las cifras anteriores muestran que es posible evitar semejante despropósito en nuestro medio.

Ligada a la disminución de la cesárea primaria, está la reducción en las tasas de cesárea por cesárea previa, o cesárea iterativa, que disminuyó de $28.5 \%$ (96/339) en el segundo período, hasta $10.9 \%(132 / 1.300)$ en el tercero. Dicho de otra manera, la cesárea primaria mostró una disminución del 72\% al tiempo que la cesárea iterativa disminuyó un 64\%. No registramos en este estudio la proporción de pacientes a quienes se ofreció la opción de un parto vaginal después de cesárea. Esta es una práctica usual en nuestro servicio como que la oferta y realización del parto vaginal cuando hay antecedentes de una a más cesáreas previas, aporta un segmento importante a la reducción de las tasas de la intervención. Es una variable que tendremos que medir de aquí en adelante, pero es un hecho conocido que solo una quinta parte de las pacientes a quienes se les ofrece aceptan este procedimiento. Por esa razón, creemos que debe evitarse en lo posible la cesárea iterativa, pero el mayor aporte descansa en la filosofía que apunta hacia la disminución de la tasa de cesárea primaria (14).

Encontramos también una notable disminución en la frecuencia con la cual se hacía un diagnóstico de desproporción cefalopélvica (DCP) antes de la iniciación del trabajo de parto. La DCP es un evento cuya frecuencia oscila entre el 1.5 y el $3.0 \%(5,15,16)$ Y cuyo diagnóstico solo puede realizarse durante el período expulsivo del parto, a pesar de los intentos para lograr un diagnóstico de la misma, antes del parto (17) que no han podido ser validados al estudiar su eficacia (18). Esta supuesta indicación, que durante el primer período de nuestro trabajo alcanzó la inverosímil frecuencia de $43.6 \%$, casi la mitad 
Cuadro 3

TASAS DE CESAREA POR INDICACION EN LOS PACIENTES MPP Y POS

\begin{tabular}{|l|c|c|c|}
\hline \multicolumn{1}{|c|}{ INDICACION } & MPP & POS & Jacksonville (9) \\
\hline Distocia & $8.1 \%(6 / 74)$ & $6.0 \%(68 / 1.116)$ & $2,90 \%$ \\
Cesárea previa & $43.2 \%(32 / 74)$ & $8.8 \%(100 / 1.126$ & $3,30 \%$ \\
Sufrimiento Fetal & $4.0 \%(3 / 74)$ & $3.4 \%(38 / 1.126)$ & $1,50 \%$ \\
Presentación podálica & $4.0 \%(3 / 74)$ & $2.6 \%(29 / 1.126$ & $0,80 \%$ \\
Solicitud materna & $16.2 \%(12 / 74)$ & - & - \\
\hline
\end{tabular}

de los diagnósticos de distocia, bajó aun 18.7\%, cifra que sigue siendo alta, pero que permite esperar una mayor reducción en el futuro. El RR para cesárea con este diagnóstico anteparto, fue 0.38 (IC95\%, 0.16-0.87), o sea una disminución del 62\%. (Figuras 3 y 4 )

Las diferencias encontradas en las características clínicas de los 2 últimos grupos estudiados, muestran un aumento estadísticamente significativo tanto en la gravidez, que tuvo un valor promedio de $1.68+0.06$ entre 1996-97 $\mathrm{y}$ de $2.05+0.06$ entre 1998-99, $\mathrm{p}=0.0001$, como en la paridad de las pacientes, que subió de $0.15+0.03$ en el segundo grupo, hasta $0.48+0.06$ en el tercero, $\mathrm{p}=0.00002$ (Cuadro 1). Este resultado indica que se intervinieron menos primigravidas y por lo tanto, puede ser un efecto de la significativa reducción en las tasas de cesárea primaria, que como vimos bajó desde $58.9 \%$ en el segundo periodo hasta $10.7 \%$, una reducción de 48.2 puntos porcentuales, en el tercer periodo.

Las indicaciones más frecuentes para las pacientes del POS siguieron siendo las mismas que describimos en nuestro primer trabajo: cesárea previa, distocia, sufrimiento fetal y presentación podálica, en ese orden. La variación se dio en sus tasas (Cuadro 2). Cuando en 1996-97 se realizó un $28.3 \%$ de cesáreas con la indicación de cesárea previa, en 1998-99 esta cifra disminuyó a un significativo 10.7\%; sin embargo, es 3 veces más alta que la informada en la literatura $(14,19,20,21,22)$. Lo mismo es cierto para el diagnóstico de distocia, que disminuyó a la mitad, pero es también 3 veces mayor que en el resto del mundo $(5,15,16)$

Figura 5

CAMBIOS EN LAS TASAS DE CESAREA EN PACIENTES USUARIAS DE MEDICINA PREPAGADA Y PARTICULAR (MPP)

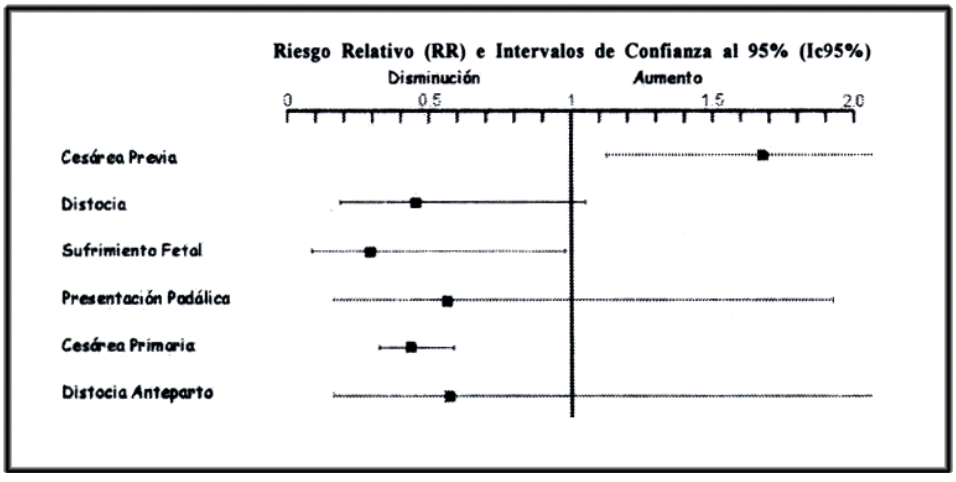

para el de sufrimiento fetal, que disminuyó también a la mitad pero es el doble de lo informado en literatura médica $(23,24)$ y para el de cesárea por presentación podálica, que disminuye también casi a la mitad, pero que resulta también 3 veces más alto que las cifras informadas en otras latitudes $(5,6)$.

En el grupo de las pacientes tributarias de MPP, la tendencia es la misma, pero la magni tud de la disminución de las tasas es muy diferente (Figura 4), y además aparece una indicación que ocupa el segundo lugar en frecuencia, que es la cesárea por solicitud materna. Estas diferencias puede deberse a razones diversas, entre las cuales podríamos anotar, la muy baja oferta de parto vaginal después de cesárea y su bajo consentimiento por las pacientes de este grupo, y la aceptación indiscriminada de la solicitud materna de operación cesárea, sin informar adecuadamente a las pacientes sobre los riesgos y los beneficios del parto y la cesárea (25). Es común justificar esta actitud en nuestro medio, invocando el principio médico de la autonomía de las pacientes, que establece que ellas tienen derecho a decidir frente a las conductas médicas, pero olvidando el principio de beneficencia, que establece que debe evitarse hacer daño. Y está bien establecido que la cesárea tiene una mortalidad materna varias veces mayor que el parto, como ampliaremos más adelante.

Estos resultados muestran como las estrategias aplicadas en nuestro servicio, se asocian con resultados tales como: a) la disminución, casi eliminación, del diagnóstico de cesárea anteparto, haciendo énfasis en un mayor conocimiento obstétrico sobre el diagnóstico y manejo de esta patología; b) la reducción de la práctica de la cesárea previa, mediante el ofrecimiento de parto vaginal después de cesárea y la disminución en las tasas de cesárea primaria y c) el diagnóstico más objetivo del sufrimiento fetal, insistiendo en la correcta interpretación de los trazados de frecuencia cardíaca fetal y eliminando terminologías que inducen a una cesárea injustificada, como: desaceleraciones profundas, bradicardias de muy corta duración y sin manejo de sus posibles causas, pérdida de la variabilidad de la línea de base en registros de 5 a 10 minutos de duración, entre otras. La disminución en la tasa de cesárea en presentaciones podálicas, parece deberse más a un aumento en la atención del parto vaginal en algunos casos de embarazo multifetal, ya que nuestra norma sigue privilegiando la cesárea en embarazos con feto único. Además, el número de casos con este diagnóstico es muy bajo, lo que le quita peso a conclusiones más definidas. 
Hemos descrito pues resultados muy alentadores, cuya explicación se asocia con las nuevas intervenciones aplicadas en nuestra clínica. La discusión semanal conjunta de la indicación de las cesáreas realizadas, resultó ser una estrategia válida. Creemos que cuando se da una discusión abierta y objetiva no puede justificarse, por ejemplo, un diagnóstico anteparto de distocia, ya que tal vez con la única excepción de la presencia de fetos con peso calculado por encima de 4.000 gramos $(26,27)$, tal conducta no puede basarse en ninguna evidencia científica, ni tampoco justificarse como práctica de una obstetricia defensiva frente a posibles problemas legales.

Así mismo, la apertura a las pacientes del POS y su atención del parto normal por médicas generales, son estrategias que también tienen que ser consideradas al analizar nuestros resultados. La menor presión que ejercen tanto las embarazadas como las médicas generales, sobre la exigencia de una operación cesárea sin ninguna otra razón que la solicitud materna, se traduce en bajas tasas de intervención, como se ha descrito en otros países en donde las embarazadas normales son atendidas por obstetrices, bajo supervisión médica, $(28,29,30,31,32,33$, 34, 35), independientemente del plan de salud al cual pertenezcan.

Aún no hemos aplicado a nuestros datos métodos de análisis que nos permitan establecer con mayor precisión la intervención, o las intervenciones específicas directamente relacionadas con los efectos obtenidos sobre las tasas de operación cesárea. Pero tal vez la razón más importante para explicar estas diferencias, es que las pacientes tributarias del POS y las de la MPP, constituyen dos poblaciones distintas por su nivel económico, que hace más exigentes a estas últimas y más condescendientes a sus médicos. Podría decirse entonces, que aunque la tendencia en la disminución de las tasas de cesárea es comparable en ambos grupos y que la disminución en las tasas de cesárea, cesárea previa y sufrimiento fetal agudo, son estadísticamente muy significativas, no lo son tanto desde el punto de vista clínico, ya que deberían ser similares en aras de un ejercicio razonable de una sola obstetricia y no de obstetricias distintas, como lo muestran estos resultados. Una demostración contundente de esta afirmación es que la tasa de cesárea iterativa disminuyó en las pacientes del POS, cuando en contraste, aumentó en las pacientes de MPP. Podría decirse entonces, que paradójicamente las pacientes del POS están siendo mejor atendidas desde el punto de vista científico que las de MPP y también, que este hecho puede estar relacionado con la atención del parto normal por médicas generales, con supervisión especializada. Esta última estrategia merece, a la luz de nuestros resultados, un estudio aleatorizado y controlado, dada su indiscutible trascendencia.

No es posible estudiar variables como la mortalidad perinatal en las tres cohortes estudiadas, ya que el número de casos es insuficiente para ese propósito y por esa razón no se incluyó como una variable de salida. Pero es muy importante destacar como se plantea con mucha frecuencia en nuestro medio que la cesárea hace parte de una estrategia de medicina defensiva, frente a las exigencias de la medicina socializada proveniente de planes de MPP o del POS y a las demandas por mala práctica. Se acepta entonces que la cesárea produce resultados perinatales superiores al parto vaginal. Sin embargo, nuestros resultados muestran lo contrario, si consideramos que el número de partos vaginales aumentó desde 109 en el segundo periodo a 910 en el tercero y que ese resultado no afectó negativamente los resultados perinatales medidos a través de la hospitalización de los recién nacidos en la UCIN. La verdad es que la proporción de hospitalizaciones en esa Unidad en relación con número de partos, disminuyó significativamente (Figura 4), dejando en claro que la disminución de la tasa de cesárea y por ende, el aumento significativo de los partos vaginales, se asoció con una clara y significativa disminución de la morbilidad neonatal.

Finalmente, quiero puntualizar que la atención del parto normal por personas diferentes a los especialistas, no debe significar un regreso a la atención obstétrica empírica, sino que al contrario, debe aceptarse como un llamado a la atención más segura de las embarazadas, siempre y cuando haya una supervisión permanente por personal especializado y existan facilidades para aplicar toda la tecnología disponible para el manejo de los casos de alto riesgo y las emergencias obstétricas. Pero dentro de esa concepción científica y tecnológica, no puede dejar de tenerse en cuenta que aún en los países más desarrollados del mundo y en servicios de excelencia, la mortalidad por cesárea es nueve a diez veces mayor que en el parto vaginal (36, 37, 38, 39), y que en los países subdesarrollados esta mortalidad puede ser muchas veces más alta (40). En nuestro servicio hemos tenido dos casos de mortalidad materna, una cifra ciertamente muy alta; y los dos casos ocurrieron en embarazadas a quienes se les practicó una operación cesárea.

\section{BIBLIOGRAFIA}

1. Coba, E. Tasas de operación cesárea en la Fundación Clínica Valle del Lili: ¿Es posible reducirlas? Colombia Med 1998; 29: 53-61.

2. Bottoms SF, Rosen MG, Sokol RJ. The increase in the cesarean birth rate. N Eng J Med 1980; 302: 559-63.

3. Brown MC. An audit on caesarean section rates in a maternity district. Br J Obstet Gynecol 1983; 90: 283-84.

4. Myers SA, Gleicher NA. A succesful program to reduce cesarean section rate. N Eng J Med 1988; 319: 1115-17.

5. Francome C, Savage W. Caesarean section in Britain and the United States. $12 \%$ or $24 \%$ : is either the right rate? Soc Sci Med 1993; 37: 1199-218.
6. Lidegaard O, Jensen LM, Weber T. Technology use, cesarean section rates and perinatal mortality at Danish maternity wards. Acta Obstet Gynecol Scand 1994; 73: 204-5.

7. Nielsen TF, Olavson PO, lngemarsson 1. The cesarean section in Sweden: the end of the rise. Birth 1994; 21: 34-8.

8. Sánchez-Ramos L, Kaunitz AM, Peterson HB, Martinez-Schnell B, Thompson RJ. Reducing cesarean section rate at a teaching hospital. Am J Obstet Gynecol 1990; 163: 1081-88.

9. Easterbrook PhJ, Berlin JA, Gapalan R, Matthews DR. Publication bias in clinical research. Lancet 1991; 337: 867-72.

10. CLASP: a randomized trial of low dose aspirin for the prevention and treatment of preclampsia among 9.364 pregnant women. 
(CLASP: Collaborative Low dose Aspirin Study in Pregnancy) Collavorative Group. Lancet; 343: 619-29.

11. Cobo E, Conde-Agudelo A, Delgado J, Canabal H, Congote A. Cervical cerclage: an alternative for the rnanagement of placenta previa? Am J Obstet Gynecol 1998; 179: 122-5.

12. Lagrew DD, Morgan MA. Decresing the cesarean section rate in a private hospital: Success without mandated clinical changes. Am J Obstet Gynecol 1996; 174: 184-91.

13. Studnicki J, Remmel R, Campbell R, Wernwer DC. The impact of legislative imposed practice guidelines on cesarean section rates: The Florida experience. Am J Med Qual 1997; 12: 62-8.

14. Kline J, Arias F. Analysis of factors determining the selection of repeated cesarean section or trial of labor in patients with histories of prior cesarean delivery. J Reprod Med 1993; 382: 8992.

15. O'Driscoll K, Jackson RJA, Galleher JT. Active management of labor and cephalopelvic disproportion. J Obstet Gynaecol Br Commonwlth 1970; 77: 385-9.

16. O'Driscoll K, Foley M, MacDonald D. Active rnanagement of labor as an alternative to cesarean section for dystocia. Obstet Gynecol 1984; 63: 48590.

17. Thurnan GR, Hales KA, Morgan MA. Evaluation of the fetal-pelvic relationship. Clin Obstet Gynecol 1992; 35: 570-81.

18. Ferguson J, Newberry Y, Turkheirner E, De Angelis G, Finnerty Y, Agawal $\mathrm{S}$. The fetal-pelvic index does not predict fetal-pelvic disproportion. Soc Perinat Obstetricians. Annual Meeting. Miami, USA. Feb 4-7, 1998.

19. Porreco RP, Meir FR. Trial of labor in patients with multiple cesarean section. Am J Obstet Gynecol 1983; 28: 770-2.

20. Flarnrn BL, Newrnan LA, Thornas SI, Falcon D, Yoshida MM. Vaginal birth after cesarean section delivery. Results of a 5- year multicentre collaborative study. Obstet Gynecol 1990; 76: 750-5.

21. Asakura H, Myers SA. More than one previous cesarean delivery. A 5-year experience with 435 patients. Obstet Gynecol 1995; 85: 924-9.

22. Odeh M, Tarazova L, Wolfsorn M, Oettinger M. Evidence that women with a history of cesarean section can deliver twins safely. Acta Obstet Gynecol Scand 1997; 76: 663-6.

23. Parer JT, Livingstone EG. What is fetal distress? Am J Obstet Gynecol 1990; 162: 1421-7.

24. Zuspan FP, Quilligan EJ, Iams JD, van Geijn HP. Predictors of intraparturn fetal distress: the role of electronic fetal monitoring. Am J Obstet Gynecol 1979; 135: 287-91.

25. Chevemak F, McCullough. An ethically justified algorithm for offering, recommending, and performing cesarean delivery and its application to managed care practice. Obstet Gynecol 1996; 87: 302-5.

26. Kolderup LB, Laros RK Jr, Musci TJ. Incidence of persistent birth injury in macrosomic infants: association with of delivery. Am J Obstet Gynecol 1997; 177: 37-41.

27. Gregory KD, Henry OA, Ramicone E, Chan LS, Platt LD. Maternal and infant complications in high and normal weight infants by method of delivery. Obstet Gynecol 1998; 179: 507-13.

28. Van Alten D, Eskes M, Treffers PE. Midwifery in the Netherlands. The Wormerveer study, selection, mode of delivery and infant morbidity. Br J Obstet Gynaecol 1989; 96: 656-62.

29. Rockenschaub A. Technology free obstetrics at the Semmelweiss Clinic. Lancet 1990; 335: 977.

30. Levy B, Wilkinson R, Marine w. Reducing neo natal mortality rate with nurse midwives. Am J Obstet Gynecol 1971; 109: 50-58.

31. International Federation of Gynaecology and Obstetrics and the International Conference of Midwives. Maternity Care in the World, 1976, $2^{\text {nd }}$ edition. FIGO/ICM

32. Barrington E. Midwifery in Canada. Toronto: New Canada Publications. 1985.

33. Blondel B, Pusch D, Schmidt E. Some characteristics of antenatal care in 13 European countries. Br J Obstet Gynaecol 1985; 92: 565-8.

34. Raisler J. Improving pregnancy outcome with nurse-midwifery care. J Nurse Midwifery 1985; 30: 189-91.

35. Blanchette H. Comparison of obstetric outcome of a primary-care access clinic staffed by certified nurse-midwives and a private practice group of obstetricians in the same community. Am J Obstet Gynecol 1995; 172: 1864-71.

36. Hall MJ. Commentary: confidential enquire into maternal death. Br J Obstet Gynecol 1990; 97: 752-3.

37. 37 Lifford RJ, van Coeverden de Groot HA, Moore PJ and Bingman P. The relative risk of cesarean secttion (antepartum and elective) and vaginal delivery: a detailed analysis to exclude the effects of medical disorders and other acute pre-existing physiological disturbances. Br J Obstet Gynecol 1990; 97: 883-93.

38. Department of Health, Welsh Office Scottish Home and Health Dept. Department 01 Health and Social Sciences Northern /reland Report on Confidential /nquiries into maternal deaths in the United Kingdom 1985-87. Her Majesty 's Stationery Office. London 1991.

39. Schuitmaker N, van Roosmalen J, Decker G, van Dongen P, van Geijn H, Gravenhorst JB. Maternal mortality after cesarean section in the Netherlands. Acta Obstet Gynecol Scand 1996; 87: 302-5.

40. Belitzky R. El nacimiento por cesárea hoy. Salud Perinatal. Boletín del Centro Latinoamericano de Perinatología y Desarrollo Humano (CLAP) 1989; 3: 101. 